\title{
Measurement and recording during intensive patient care
}

\author{
Percy Cliffe
}

\author{
Department of Clinical Measurement, Westminster Hospital, London
}

ANY PROBLEM in clinical measurement involves an analysis of the medical requirements in order to assess what information is to be obtained and how it is to be used.

This approach is quite general. The Clinical Measurement Department at Westminster Hospital is a service department organized under the National Health Service and is concerned with a wide variety of clinical measurements ranging from cardiac investigations, lung function testing, surgical monitoring, haemodialysis, intensive care, etc., carried out in close collaboration with the clinicians concerned.

In each instance, it is necessary to decide what information is required to solve the particular medical problem.

In illustration, I would like to take one example of intensive care, namely of patients who have undergone cardiac surgery using profound hypothermia. Clearly measurements on such patients, following thoracotomy with involvement of the heart and lungs, is designed to detect and offset post-operative complications.

Consider some of the important factors. Blood loss and impaired cardiac action can lead to the features of shock, that is, to a state of circulatory failure characterized by systemic arterial hypotension and peripheral vasoconstriction. A low cardiac output and stroke volume may result from the operative procedures in the heart itself. Again, a time lag in fluid replacement or a failure of the peripheral circulation results in the central venous pressure being insufficient to fill the right side of the heart. By contrast, tamponade or a failing heart result in a low output with a raised venous pressure.

The response to a low cardiac output is a compensatory vasoconstriction which assists circulation through the carotid and coronary vessels but which is of temporary benefit, for reduced perfusion of tissues leads to hypoxia and metabolic acidosis.

These changes are associated with and complicated by the impairment of respiratory exchange. Inadequate oxygenation of arterial blood with reduction in blood oxygen tension accompanies alveolar hypoventilation or hypoventilation from respiratory depression with low tidal and minute volumes. Maldistribution between ventilation and perfusion can occur from collapse, accumulation of secretions, haemorrhage and so on.

This brief statement of the situation immediately suggests which items of information are required.

Table 1 shows the parameters currently being measured. Blood loss is measured separately from the

TABLE 1

Table of measurements

\begin{tabular}{ll}
\hline \multicolumn{1}{c}{ Continuous } & \multicolumn{1}{c}{ Intermittent } \\
\hline ECG & Blood loss \\
Pulse rate & Arterial oxygen saturation \\
Systolic pressure & Venous oxygen saturation \\
Diastolic pressure & Respiration rate \\
Right atrial pressure & $\begin{array}{l}\text { pH, PcO 2, Po } 2 \\
\text { Temperatures }\end{array}$ \\
& Bicarbonate \\
\hline
\end{tabular}

mediastinum and pericardium and from the pleural cavities if these have been opened during surgery. The pericardial drain is essential and, if functioning properly, avoids tamponade. In addition to blood loss, the ECG is observed and the pulse rate is derived from it.

Arterial pressure is recorded by electromanometry via a disposable Guest cannula introduced into the radial artery by Seldinger technique or by direct cut down. The artery is preserved and the pulse is palpable soon after removal.

Right atrial pressures are recorded similarly via a direct catheter into the atrial appendage using a disposable epidural catheter with side holes. It can be left in place for 2-3 days without the slightest fear of haemorrhage on removal.

If a mean pressure is being recorded, it is necessary to check that a rise in pressure is not due to the catheter having slipped into the right ventricle-a fact immediately obvious by the record switching to the pulsatile waveform.

It has been found that the right atrial pressure is an invaluable parameter in fluid replacement. It is measured at RA level-not referred to the sternal angle-and decreasing pressure indicates undertransfusion and a rise indicates over-transfusion, tamponade or right heart failure.

For relatively simple repairs, the atrial pressure 
aimed at is about 3-5 $\mathrm{cmH}_{2} \mathrm{O}$, for Fallot's 7-10 cm and valve replacements about $10-15 \mathrm{cmH}_{2} \mathrm{O}$ for $12 \mathrm{hr}$ or more.

During profound hypothermia, citrated blood primes the machine and can result in considerable haemodilution. A positive balance of 3 litres or more may be necessary in the first $12 \mathrm{hr}$. The administration of citrate tends to produce an alkalosis but does not harm the patient in any way after correction of low calcium ion concentration.

A rise of RA pressure to $20 \mathrm{cmH}_{2} \mathrm{O}$ or more indicates tamponade or heart failure and the pericardial drain has proved of value here. In this situation, the cardiac output is of obvious value, and the arterio-venous oxygen saturation difference by the Kipp haemoreflector is used as an indicator of cardiac output. With a normal arterial saturation and basal metabolic conditions, a venous saturation of less than $50 \%$ is likely to indicate serious heart failure.

However, the arterial saturation may be far from normal and as these patients are recovering from hypothermia, their metabolism may be depressed.

Anoxia can lead to death from ventricular fibrillation and $\mathrm{PO}_{2}$ and $\mathrm{PCO}_{2}$ are the most sensitive guides to adequacy of respiratory exchange. Although respiration rate, tidal volume and inflation pressure may be determined with patients on controlled respiration, the $\mathrm{Po}_{2}$ and $\mathbf{P C O}_{2}$ are still essential for the management of the ventilator.

Only respiratory rate is measured for the patient breathing spontaneously, and the $\mathrm{PO}_{2}$ is thus the only reliable guide to adequate arterial oxygenation in these cases.

Under conditions of respiratory and circulatory disturbance the acid-base situation must be assessed and the $\mathrm{pH}, \mathrm{PCO}_{2}$, bicarbonate and base excess are determined at frequent intervals by the Astrup apparatus.

Temperatures, usually rectal and axillary are measured by thermocouple probes.

Fig. 1 illustrates the intensive care record of a patient following replacement of mitral and aortic valves by Starr Edward prostheses.

Here we have a patient returning cold to the ward with a rectal temperature below $36^{\circ}$, hypovolaemic with a falling blood pressure and arterial saturation, a low venous pressure and a rapid, fluctuating pulse.

The cardiac output indicated by the $\mathrm{A} / \mathrm{V} \mathrm{O}_{2}$ difference was falling, he was becoming increasingly acidotic with falling $\mathrm{pH}$, low bicarbonate and rising $\mathrm{PCO}_{2}$.

Blood was administered in large amounts on the indication of the venous pressure and after $2 \mathrm{hr}$ the blood pressure began to rise. By now the arterial saturation was $92 \%$ and the $\mathrm{PCO}_{2}$ was approaching $60 \mathrm{mmHg}$ and intermittent positive pressure respiration was instituted. The patient was tried on spon- taneous respiration after $12 \mathrm{hr}$, when his general $\stackrel{\frac{\$}{2}}{\circ}$ condition was considerably improved and monitor- $C$. ing was discontinued after $16 \mathrm{hr}$.

Fig. 2 shows similar sequences following replace- $\stackrel{2}{\rightarrow}$ ment of an aortic valve. The patient was breathing spontaneously. Again a falling venous pressure in the $\frac{\bar{c}}{\bar{c}}$. first $2 \mathrm{hr}$ increased with fluid replacement. The $\overrightarrow{\mathbb{\sigma}}$

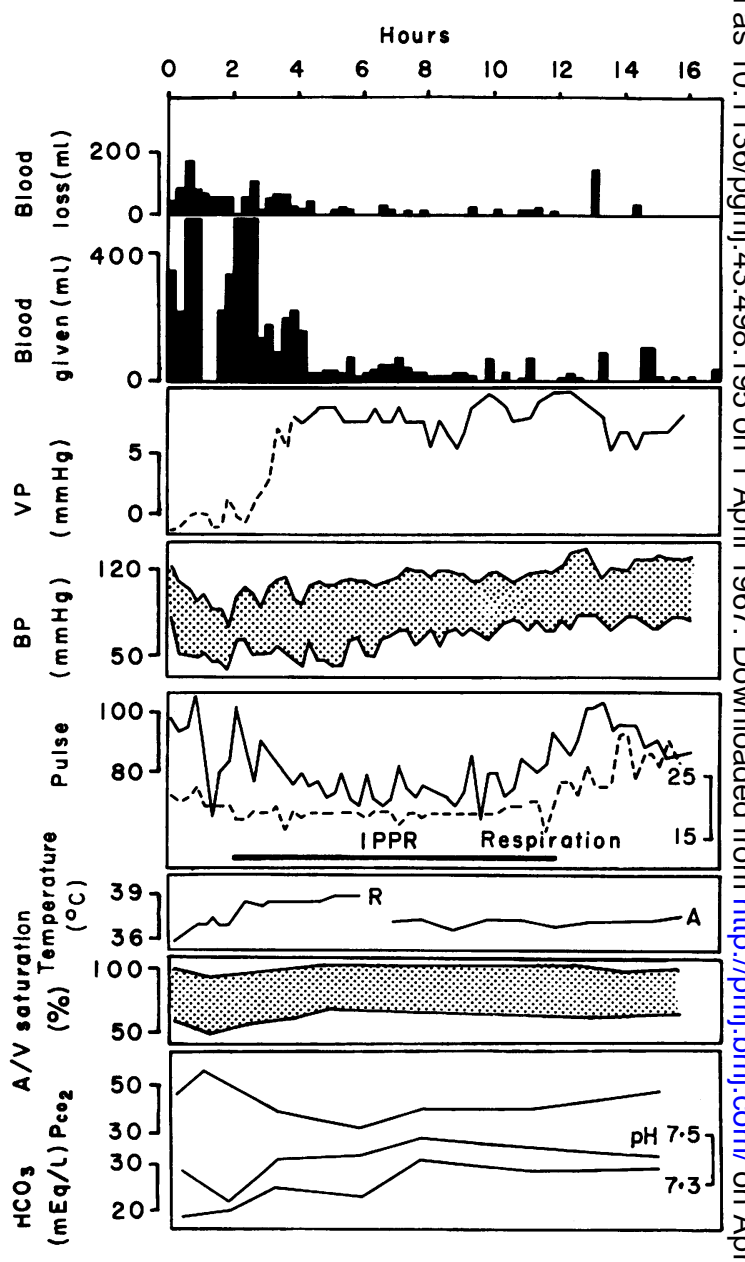

Fig. 1. Post-operative recordings following replacement of aortic and mitral valves.

blood pressure remained steady and cardiac output $\tilde{O}$ rose and adequate arterial oxygenation was main- $\mathbb{\omega}$ tained and an initially high $\mathrm{PCO}_{2}$ gradually decreased.

\section{Additional measurements}

Reference has been made to the value of a more or less direct measurement of cardiac output and it $\frac{T}{5}$ is proposed to apply dye-dilution techniques to our present estimations using indocyanine green in $\stackrel{\mathbb{P}}{\stackrel{\oplus}{\circ}}$ association with one of the commercial special pur- $\stackrel{\square}{\square}$ pose computers. This has the added advantage of $\bar{\gamma}$ detecting intracardiac shunts post-operatively and 
might sometimes be useful in the differential diagnosis of a decrease in arterial saturation.

Measurements of respiratory volumes are difficult and although the impedance pneumograph is a possibility it has associated problems, even if it were calibrated by spirometry in theatre immediately following operation.

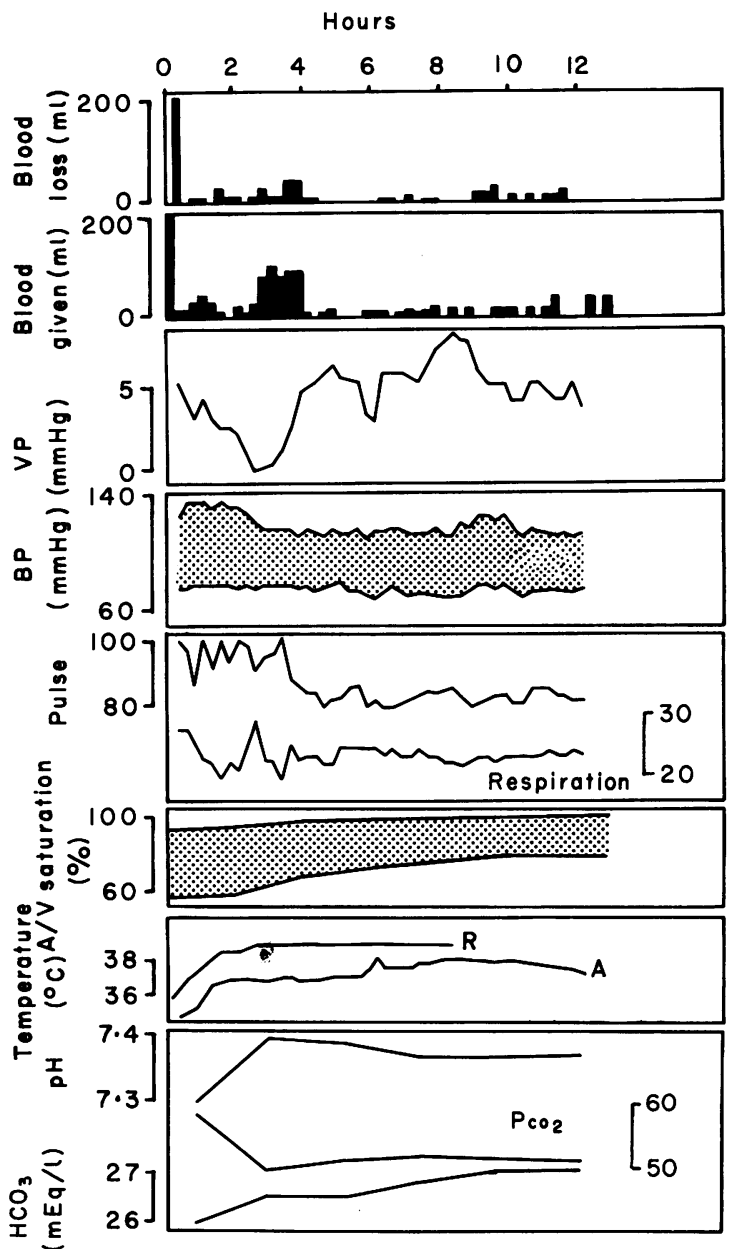

FIG. 2. Post-operative recordings following replacement of aortic valve.

Another parameter of interest is the left atrial pressure following left-heart surgery. It would give a direct measure of failure of $\mathrm{LV}$ function and would provide a valuable means of distinguishing heart failure from causes in the LV, the pulmonary circulation and the right ventricle. A persistent high LA pressure raises the question of temporary assisted circulation of the left heart.

\section{Recording of information}

The present method of collecting information involves essentially three time scales.
(1) Waveforms of the relatively rapid phenomena such as ECG and arterial pressure are observed on an oscilloscope.

(2) A multichannel pen recorder charts arterial and venous pressures, together with ECG and pulse rate. A fairly slow paper speed of about $3 \mathrm{~cm} / \mathrm{min}$ is used. This is sufficient for detecting trends, such as the response of atrial pressures to blood replacement or to changes in arterial pressure. The multichannel tracings act as an early warning system and at any particular time, the previous $3 \mathrm{~min}$ of tracing can be seen.

(3) A large pre-printed chart $2 \mathrm{ft} \times 2 \mathrm{ft} 6$ in. is employed on which all the results are entered by hand at quarter-hourly intervals. This large chart provides the main information of response to treatment and drugs, and procedures are entered on it, together with the intermittent determinations of arterial and venous oxygen saturation, $\mathrm{Po}_{2}, \mathrm{pH}$, $\mathrm{PCO}_{2}$, bicarbonate and respiratory rate.

Consideration is being given at present to further developments in presentation. Fig. 3 illustrates the

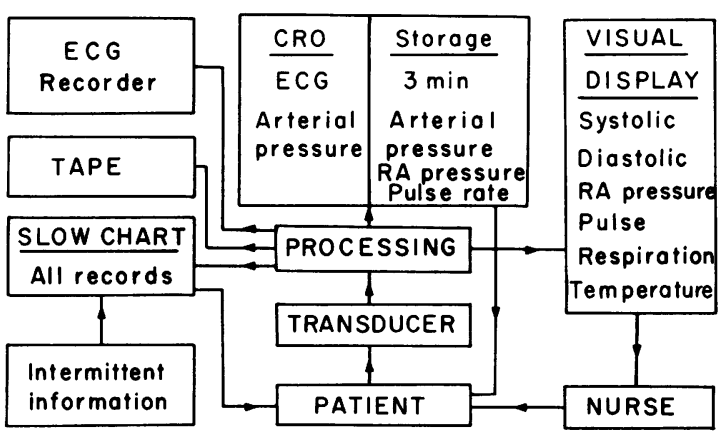

FIG. 3. System of presenting information during postoperative measurements.

general arrangement. Information from the patient is sensed by transducers and processed in such a way that it can be presented in several visual displays. For example, the 3-min interval of information for early warning might be viewed on an image storage tube, particularly of the type in which two portions of the screen may separately store the trace. Such a device suitably switched can retain the last items of information when the time-base flies back and the trace repeats. The large chart is extremely valuable and forms the main presentation on which decisions are taken during the intensive care period, in relation to the clinical condition of the patient.

Devices are being examined which will produce this chart more or less automatically. For example, a multichannel potentiometric recorder might be used or a chart printer, similar to that of the 'Monitron' equipment but modified so that printing occurs 
over the top half of the chart leaving the rest for entering results by hand. Alternatively an $X-Y$ plotter with a suitable switching sequence between adjacent channels might serve the purpose. Large plotters, for example $30 \times 30$ in., are commercially available and would leave adequate space for plotting the intermittently generated results.

For a visual display, which is extremely valuable to the nursing staff, the 'Monitron' type appears to have great advantages. Information is presented vertically as in a mercury thermometer and the device is illuminated for use in a darkened ward.

Feed-back of information to the patient from these various presentations is being continuously effected by the medical and nursing staff.

\section{Techniques}

One or two words on technique.

\section{Blood pressure}

Intravascular catheters are used with electromanometers, which are usually Statham strain gauges. The acrylic head is removed and replaced by a tap system, illustrated by Fig. 4 . A needle valve

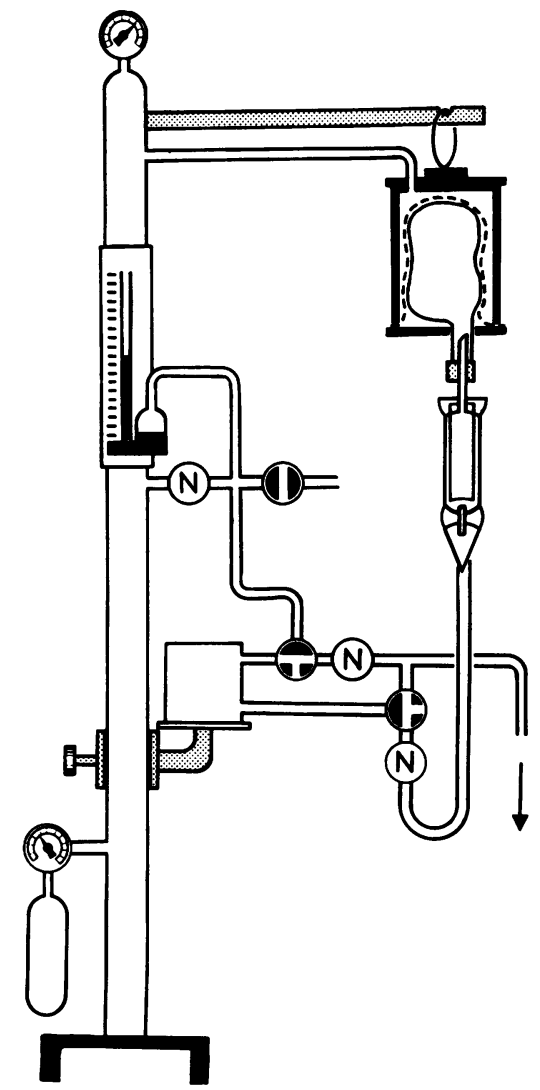

Fig. 4. Blood pressure transducer and control unit. allows control of the drip rate, and a pressure cali $\stackrel{\Phi}{\stackrel{2}{2}}$ bration can be applied without altering the drip.Damping may be adjusted by means of a secons needle valve.

These transducers are used in combination with the control unit resembling a transfusion stand as shown in Fig. 4. This unit pressurizes the drip set via plastic bag. If the seams of the bag are imperfect of if the bag empties, oxygen under pressure cannots enter the giving set. The system allows a constan 5 pressure to be applied to the bag as it empties.

The control unit provides a calibrating pressure for the transducer. The pressure can be set up on के mercury manometer. The transducer can be raised or lowered so that any hydrostatic pressure difference between the transducer and the point of measure ment is eliminated.

\section{Cardiac output}

During the past 2 years, the Gilford cuvette has been found satisfactory for the measurement of cardiac output using indocyanine green in combina $\rightarrow$ tion with a general purpose analogue computer to․ analyse a dye dilution curve.

Fig. 5 shows a calibration for a series of blood $\vec{\circ}$

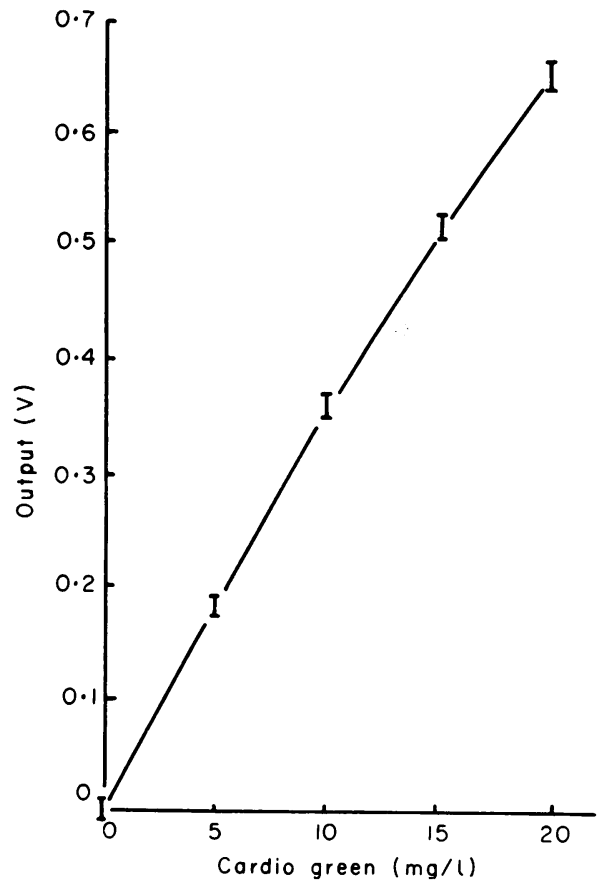

Fig. 5. Calibration of Gilford cuvette with blood containing indocyanine green dye and varying haematocrits from $32 \%$ to $56 \%$.

samples containing indocyanine green. When the haematocrit was varied between $32 \%$ and $56 \%$, theo variations produced a series of parallel calibration lines, and in use only the zero has to be adjustedo 
before each dye curve is transcribed. The system is essentially linear, and drift may be reduced to approximately $1 \%$ of the peak height of a curve over a 6-hr period.

The principle of computer calculation is shown in Fig. 6. The computer uses the height and separation

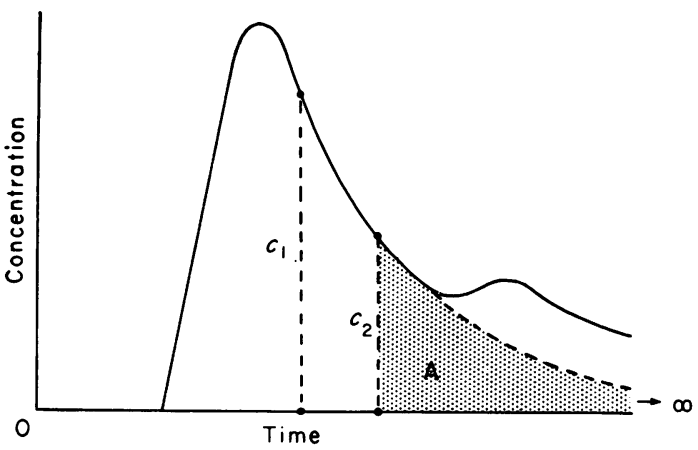

Fig. 6. Principle of cardiac output calculations using analogue computer.

of any two ordinates $C_{1}$ and $C_{2}$ on the downslope to calculate the area $A$ of the extrapolated curve beyond $C_{2}$. This area is added to that from zero time up to $C_{2}$ to give the total area, when

$$
\text { Cardiac output }=\frac{\text { Dye dose }}{\text { Total area }}
$$

\section{Predictive monitoring}

Caceres (1963) and Pipberger (1965) have shown how electrocardiograms can be analysed and diagnosed by digital computer.

Caceres (1966) has shown that measurement of all the amplitudes and intervals of the ECG can be effected in about $3 \mathrm{sec}$. This analysis can be ongoing in line, i.e. the results can be written out as a graph or plotted against time with a pen recorder so that changes in the ECG pattern can be followed with great precision.

Sayers (1966) has pointed out that methods are available for assessing the extent and nature of irregularities which might occur in a series of cardiac cycles. In collaboration with him, work is currently being carried out at Imperial College by Dr J. P. Blackburn who has analysed a record of 20,000 successive ECG complexes of a patient with atrial fibrillation. This patient had an episode of supraventricular tachycardia lasting a few minutes, although this incident passed unnoticed by patient or nurse.

The record is being examined for the variation in beat-to-beat interval to see if increasing runs of short beats built up before the onset of the tachycardia. Plots of expectation density function and scaled histograms are being employed. This experiment utilizes only a single parameter of the ECG but, as Caceres has shown, many ECG parameters can be derived and all these could be subjected to analyses of this type, the results of which could be cross-correlated with other simultaneous physiological recordings for the purposes of predictive monitoring.

It cannot be doubted that methods such as these, used on line, will avert many of the disasters which may threaten the intensive care patient of the near future.

\section{Acknowledgments}

The author gratefully acknowledges the advice of members of the Intensive Care team, in particular, Dr M. J. Pemberton, F.F.A.R.C.S., and Mr P. A. Cullum, F.R.C.s.

\section{References}

CACeres, C.A. (1963) Electrocardiographic analysis by a computer system. Arch. intern. Med. 111, 196.

CACERES, C.A. (1966) Report of Conference on Medical Computers, Elsinore 1966 (In press).

Pipberger, H.V. (1965) Computers in Biomedical Research (Ed. by R. W. Stacey and B. Waxman), vol. 1, p. 377. Academic Press, New York.

SAYERS, B.MCA. (1966) Lecture 'Medical Engineering in Relation to Clinical Measurement' to Societa MedicaChirurgica, Bologna.

\section{Discussion to the paper by $\mathbf{P}$. Cliffe}

ROBERTS. Dr Cliffe has spoken of recording or monitoring fourteen different physiological parameters during intensive patient care and it is clear that there must be considerable problems in taking note of and in using all this information. The measurement of, say, the beart rate is only the first link in a chain of communication which returns ultimately to the patient via one or more doctors or nurses, who can only take the appropriate action to correct changes in heart rate if the information provided by the recording system is brought to their notice at sufficiently frequent intervals. An automatic observer such as a computer could well monitor and carry out corrective action in a continuous manner with virtually no time lag between observation and action but the relative slowness of response of the human observer makes it impossible to achieve this ideal state of affairs. It is therefore necessary to decide in advance how frequently to monitor any particular parameter, or when continuous monitoring is possible, how frequently to observe and act upon the monitored information.

The use of a computer to predict changes in cardiac action was suggested by Dr Cliffe with particular reference 